\title{
Physicochemical and Phytochemical Examination of Medicinal Plants Used in Indigenous System of Medicine
}

\author{
M. K. SANTOSH,* D. SHAILA, T. CHANDRAKUMAR, \\ I. RAJYALAKSHMI and I. SANJEEVA RAO \\ Varun Herbals, 5-8-293/A, Mahesh Nagar, \\ Chirag Ali lane, Hyderabad - 500001
}

Received 2 February 2005; Accepted 9 March 2005

\begin{abstract}
The present paper deals with the physicochemical and phytochemical examination of seventy-six medicinal plants belonging to thirty-six dicot and six monocot families. These are used in indigenous system of medicine as well as local inhabitants either as single drugs or in combination, for the cure of various ailments. In physicochemical study, the parameters such as moisture content, $p \mathrm{H}$ (1\% aqueous), total ash, acid insoluble ash, water-soluble extractive and alcohol soluble extractive were carried out. The preliminary phytochemical study was done for the detection of secondary metabolites such as alkaloid, flavonoid, glycoside, phenol, saponin, resin, steroid and tannin. The preliminary phytochemical study revealed the presence of alkaloid and saponin in $68.4 \%$; flavonoid in $44.7 \%$; glycoside, phenol and steroid in $72.37 \%$; resin in $60.5 \%$ and tannin in $71 \%$ of selected medicinal plants.
\end{abstract}

Key words: Physicochemical, Phytochemical, Medicinal plants

\section{Introduction}

Indian Ayurvedic Pharmacopoeia recorded more than 300 medicinal plants that are in common use in indigenous system of medicine. It has been observed that there is a wide dissimilarity and variation in clinical results obtained by the use of crude drugs obtained from different geographical regions ${ }^{1}$. The knowledge of chemical compounds present in a plant helps the scientists to understand the mode of action of $\mathrm{drug}^{2}$.

The preliminary phytochemical screening of medicinal plants was reported by several workers ${ }^{1-6}$. The variation in phytochemical results was found among these workers.

In the present investigation out of the selected 76 plants, 63 plants are covered in the Ayurvedic Pharmacopoeia but physicochemical test limits are provided only for total ash, acid insoluble ash, water soluble extractive and alcohol soluble extractive. The acid insoluble ash values of Oryza sativa and Zingiber officinale, alcohol soluble extractive value for Trapa bispinosa and water soluble extractive value for Withania somnifera are not provided. The alcohol soluble extractive values of Ferula foetida, Holarrhena antidysenterica, Withania somnifera and Zingiber officinale are provided in Ayurvedic Pharmacopoeia using $90 \%, 60 \%, 25 \%$ and $90 \%$ aqueous alcohol respectively ${ }^{7-10}$. 
Medak district is one of the 10 districts of Telangana region of Andhra Pradesh has forests, which are of the southern tropical dry deciduous type account for $9.9 \%$ of the total geographical area. It lies between $17^{\circ} 27^{\prime}$ and $18^{\circ} 18^{\prime}$ Northern latitude and $77^{\circ} 28^{\prime}$ and $79^{\circ} 10^{\prime}$ of Eastern longitude. The rainfall during the southwest monsoon months amounts to about $84 \%$ of the annual rainfall. The average annual rainfall in the district is $896.7 \mathrm{~mm}$. Narsapur reserve forest of Medak district has a forest area of 202.70 sq. $\mathrm{km}$ and is a good source of medicinal plants. Black cotton soils are moderately alkaline with high soluble salt content and comprising of clay loams, clays and silty clays are found in Narsapur ${ }^{11}$. Narsimha Rao (1986) reported 616 taxa of angiosperms including 116 cultivated species, belonging to 395 genera and 111 families ${ }^{12}$. The local herbal drug pharmacies utilize the available raw drugs from this forest area. In the present investigation, 38 medicinal plants were collected from the same area and the remaining drugs were procured from the local market.

In view of the above facts and growing interest on plant sources for evolution of therapeutic agents, the present investigation of physico-chemical and phytochemical study of seventy-six medicinal plants belonging to thirty-six dicot and six monocot families has been taken up to determine the quality of raw drugs.

\section{Materials and Methods}

The authentic plant materials were collected from Narsapur forest, Medak district, Andhra Pradesh and identified using Flora of Medak District (Andhra Pradesh) ${ }^{11}$. Voucher specimen of the collected plants were prepared and maintained. The ingredients that were not available in and around were procured from the local market of Hyderabad, Andhra Pradesh. The respective plant parts were shade dried and finely powdered using laboratory blender and fine powder was used for further studies.

The physico-chemical parameters such as moisture content, $p \mathrm{H}$ (1\% aqueous), total ash, acid insoluble ash, alcohol soluble extractive, water soluble extractive and preliminary phytochemical screening for the presence of alkaloid, flavonoid, glycoside, phenol, saponin, resin, steroid, tannin were carried out by following the standard procedures ${ }^{13-16}$.

\section{Physicochemical tests}

Moisture content: Weigh accurately $5 \mathrm{~g}$ of powdered material in a dry and flat petri dish. Dry the sample in an oven at $110^{\circ} \mathrm{C}$. Dry until two consecutive weighing do not differ by more than $5 \mathrm{mg}$. Calculate the loss of weight in terms of percentage.

pH (1\% aqueous): Dissolve $1 \mathrm{~g}$ of powdered sample in $100 \mathrm{ml}$ of distilled water, shake frequently, then allow it to stand for 18 hours. Filter and check the $\mathrm{pH}$ using $\mathrm{pH}$ meter.

Total ash: Weigh accurately about $2-5 \mathrm{~g}$ of dried plant material in a previously ignited and tared crucible. Ignite it by gradually heating it to $500-600^{\circ} \mathrm{C}$ until it is white. Cool in a desiccator and weigh. Calculate the content of total ash in terms of percentage.

Acid insoluble ash: To the crucible containing total ash, add $25 \mathrm{ml}$ of $\mathrm{HCl}(\sim 70 \mathrm{~g} / \mathrm{l})$, cover with watch glass and boil gently for $5 \mathrm{~min}$. Rinse the watch glass with $5 \mathrm{ml}$ of hot water and add this liquid to the crucible. Collect the insoluble matter on an ashless filter paper and wash with hot water until the filtrate is neutral. Ignite the filter paper containing insoluble matter in crucible to constant weight. Cool in a desiccator and weigh. Calculate the content of acid insoluble ash in terms of percentage.

Water-soluble extractive:Weigh accurately about $4 \mathrm{~g}$ of air-dried material in a glass stoppered conical flask. Macerate with $100 \mathrm{ml}$ of distilled water for 6hours, shaking frequently, then allow to stand for 18 hours. Filter rapidly taking care not to lose any solvent, transfer $25 \mathrm{ml}$ of the filtrate to a tared flat bottomed petri dish and evaporate to dryness on a water bath. Dry at $105^{\circ} \mathrm{C}$ for 6 hours, cool in a desiccator for $30 \mathrm{~min}$ and weigh. Calculate the content of water- soluble matter in terms of percentage.

Alcohol soluble extractive:Weigh accurately about $4 \mathrm{~g}$ of air-dried material in a glass stoppered conical flask. Macerate with $100 \mathrm{ml}$ of absolute alcohol for 6hours, shaking frequently, then allow to stand for 18 hours. Filter rapidly taking care not to lose any solvent, transfer $25 \mathrm{ml}$ of the filtrate to a tared flat bottomed petri dish and evaporate to dryness on a water bath. Dry at $105^{\circ} \mathrm{C}$ for 6 hours, cool in a desiccator for $30 \mathrm{~min}$ and weigh. Calculate the content of alcohol-soluble matter in terms of percentage. 
Phytochemical tests:

Alkaloid (Wagner's test): Acidify $1 \mathrm{ml}$ of alcoholic extract of the drug with $1.5 \%$ of $\mathrm{HCl}$ and add a few drops of Wagner's reagent. A brown precipitate indicates positive test for alkaloids.

Flavonoid: In a test tube containing $0.5 \mathrm{ml}$ of the alcoholic extract of the drug, add 5-10 drops of dilute $\mathrm{HCl}$ followed by a small piece of magnesium. Boil the solution for a few minutes. In the presence of flavonoids a pink, reddish pink or brown color is produced.

Glycoside: Dissolve a small amount of alcoholic extract of the drug in $1 \mathrm{ml}$ of water and add $1 \mathrm{~N} \mathrm{NaOH}$ solution. A yellow color indicates the presence of glycosides.

Phenols $\left(\mathrm{FeCl}_{3}\right.$ test): Dissolve a small quantity of alcoholic extract of the drug in $2 \mathrm{ml}$ of distilled water and a few drops of $10 \%$ ferric chloride solution. A blue or green color is produced indicates the presence of phenols.

Saponin: Dissolve a small quantity of alcoholic extract of the drug in $5 \mathrm{ml}$ of distilled water, shake the mixture vigorously and leave for $3 \mathrm{~min}$. Honeycomb like froth indicates the presence of saponins.

Resin: Dissolve a small quantity of ethanolic extract of the drug in $5 \mathrm{ml}$ of acetic anhydride by means of gentle heat, cool and add a drop of sulphuric acid. A bright purplish red color indicates the presence of resins.

Steroid (Liebermann - Burchard's test): To the ethanolic extract of the drug in $\mathrm{CHCl}_{3}$, add acetic anhydride followed by $1 \mathrm{ml}$ of concentrated sulphuric acid. A reddish brown ring is formed at the juncture of two layers indicates the presence of steroids.

Tannin: To the ethanolic extract of the drug, add a few drops of 5\% aqueous ferric chloride solution. A bluish black color indicates the presence of tannins.

\section{Results and Discussion}

The results of the physicochemical and phytochemical study are tabulated in Table $\mathbf{1}$ and $\mathbf{2}$. A slight variation in physicochemical results was found in 46 plants of present study with that of Ayurvedic Pharmacopoeial standard values. Among these 46 plants, the variation in total ash, acid insoluble ash, water-soluble extractive and alcohol soluble extractive was observed in $23,15,30$ and 18 selected plants respectively. The preliminary phytochemical study revealed the presence of alkaloid and saponin in $68.4 \%$; flavonoid in $44.7 \%$; glycoside, phenol and steroid in $72.37 \%$; resin in $60.5 \%$ and tannin in $71 \%$ of selected medicinal plants. The variation in phytochemical results of present study was found with that of reported ${ }^{1-6}$.

The slight variation in physicochemical and phytochemical results may be due to several factors such as different geographical conditions, edaphic factors, environmental conditions, period of cultivation and harvesting, method of collection, source of irrigation and fertilizers, age of the plant, powdering method, and extraction method.

It has been reported that the variation in physico-chemical values may be due to powdering of fibrous material of an ingredient in mixer grinder, while this fibrous material is discarded during powdering in mortar and pestle. Similarly, the total ash and acid insoluble ash were slightly higher in the mixer grinder made material as the powdered fibres contribute additional $\mathrm{ash}^{17}$.

Though, a slight variation in physicochemical parameters was observed in some of the selected plants; phytochemically they showed better test results. Apart from the physicochemical parameters provided in Ayurvedic Pharmacopoeia, the data for moisture content, $\mathrm{pH}$ value and phytochemical study evolved can be considered as viable parameters, which will go a long way for prescribing a dependable standards to the raw drugs.

Hence, this study has been carried out along with the parameters given in Pharmacopoeia to determine the quality of the raw drugs, as these are used in the preparation of Ayurvedic and Unani formulations in this region. 
Table - 1: Results of physico-chemical study

\begin{tabular}{|c|c|c|c|c|c|c|c|c|c|c|}
\hline S. No. Botanical name & Family & Part used & 1 & 2 & 3 & 4 & 5 & 6 & 7 & 8 \\
\hline 1. Abutilon indicum (Linn.) Sw. & Malvaceae & Rt. & Narsapur & 58 & 7.85 & 7.03 & $8.5^{*}$ & 0.82 & 9.65 & 3.35 \\
\hline 2. Acorus calamus Linn. & Araceae & Rz. & Market & -- & 11.49 & 4.62 & 6.52 & 0.35 & 19 & 9.5 \\
\hline $\begin{array}{l}\text { 3. Aegle marmelos Corr. } \\
4.65^{*}\end{array}$ & Rutaceae & Fr. & Narsapur & 06 & 8.12 & 5.66 & 3.87 & $3.15^{*}$ & $12.55^{*}$ & \\
\hline $\begin{array}{l}\text { 4. Aegle marmelos Corr. } \\
3.75^{*}\end{array}$ & Rutaceae & St. Bk. & Narsapur & 06 & 6.09 & 6.94 & $12.81 *$ & 0.43 & $6.0 *$ & \\
\hline 5. Aerva lanata (L.) Juss. ex. Schult & Amaranthaceae & Rt. & Narsapur & 45 & 7.59 & 5.9 & 10.93 & 1.4 & 6.55 & 2.85 \\
\hline 6. Ailanthes excelsa Roxb. & Simarubaceae & St. Bk. & Narsapur & 02 & 7.55 & 6.45 & $12.03 *$ & $0.57 *$ & 7.8 & 6.9 \\
\hline $\begin{array}{l}\text { 7. Aloe vera Tourn.ex. Linn. } \\
29.8^{*}\end{array}$ & Liliaceae & Lf.Exd. & Market & -- & 7.8 & 6.56 & 3.17 & 1.9 & $45.95^{*}$ & \\
\hline 8. Alpinia galangaWilld. & Zingiberaceae & Rz. & Market & -- & 7.08 & 5.08 & 7.46 & 1.3 & 6.0 & 4.1 \\
\hline 9. Areca catechu Linn. ex. Benth. & Palmae & Sd. & Market & -- & 9.27 & 5.83 & 2.35 & $1.81 *$ & 12.35 & 9.9 \\
\hline 10. Asparagus racemosus Willd. & Liliaceae & Rt & Narsapur & 27 & 5.01 & 8.11 & 3.23 & 0.39 & $26.05 *$ & 6.0 \\
\hline 11. Asteracantha longifolia Nees & Acanthaceae & Rt. & Narsapur & 03 & 9.86 & 6.2 & $14.16^{*}$ & $2.75^{*}$ & 9.0 & $3.34 *$ \\
\hline 12. Azadirachta indica A. Juss & Meliaceae & St. Bk. & Narsapur & 42 & 12.78 & 6.15 & 5.67 & 0.26 & 8.7 & 10.1 \\
\hline 13. Bacopa monnieri (Linn.) Pennel & Scorphulariaceae & $\mathrm{Pl}$ & Narsapur & 67 & 9.82 & 6.68 & 16.18 & 2.45 & $7.8 *$ & 6.9 \\
\hline 14. Bambusa arundinacea Willd. & Gramineae & S.C. & Market & -- & 1.09 & 8.93 & 91.45 & 87.3 & 1.55 & 0.45 \\
\hline $\begin{array}{l}\text { 15. Berberis aristata DC. } \\
2.55^{*}\end{array}$ & Berberidaceae & St. & Market & -- & 5.71 & 4.92 & $14.96^{*}$ & $6.45^{*}$ & $4.45^{*}$ & \\
\hline 16. Boerhaavia diffusa Linn. & Nyctaginaceae & Wh. Pl. & Narsapur & 17 & 6.92 & 5.37 & 13.93 & 0.57 & 19.85 & 7.8 \\
\hline 17. Carum carvi Linn. & Umbelliferae & Fr. & Market & -- & 5.89 & 4.96 & 7.85 & 0.55 & 18.35 & 7.65 \\
\hline 18. Cassia fistula Linn. & Leguminosae & Fr.P & Narsapur & 34 & 10.82 & 5.58 & $9.4 *$ & 0.46 & $7.15^{*}$ & 15.35 \\
\hline 19. Centella asiatica (L.) Urban & Apiaceae & Wh. Pl. & Narsapur & 26 & 9.64 & 6.83 & 16.3 & 3.92 & $12.65^{*}$ & 11.5 \\
\hline $\begin{array}{l}\text { 20. Cinnamomum camphora Nees } \\
\& \text { Eberm }\end{array}$ & Lauraceae & Vol. Matter & Market & -- & -- & 9.29 & -- & -- & 0.2 & 14 \\
\hline $\begin{array}{l}\text { 21. Cinnamomum tamala Nees } \\
\& \text { Eberm }\end{array}$ & Lauraceae & Lf. & Market & -- & 4.67 & 6.04 & 4.46 & 0.19 & $6.85 *$ & 8.1 \\
\hline 22. Cinnamomum zeylanicum Blume & Lauraceae & St. Bk. & Market & -- & 7.5 & 7.9 & 2.72 & 0.25 & 5.4 & 6.7 \\
\hline 23. Commiphora myrrha (Nees)Engl.B & Burseraceae & Exd. & Market & -- & 10.9 & 4.85 & 5.62 & 0.21 & 39.1 & 7.05 \\
\hline 24. Coriandrum sativum Linn. & Umbelliferae & Fr. & Market & -- & 5.93 & 8.41 & 5.46 & 0.55 & 20.9 & 10.95 \\
\hline 25. Cuminum cyminum Linn. & Umbelliferae & Fr. & Market & -- & 8.59 & 7.7 & $8.14 *$ & 0.43 & $14.5^{*}$ & 7.8 \\
\hline
\end{tabular}


Rz. Market $--$

5.75

6.1

6.7

0.6

$\begin{array}{ll}16.29 & 8.05\end{array}$

Rt. Narsapur

12.32

5.17

Contd......

\begin{tabular}{|c|c|c|c|c|c|c|c|c|c|c|}
\hline 28. Cyperus rotundus Linn. & Cyperaceae & Rz. & Narsapur & 01 & 9.98 & 8.24 & 5.62 & 3.13 & 13.1 & 5.75 \\
\hline 29. Desmostachya bipinnata Stapf. & Gramineae & Rt. & Narsapur & 20 & 2.25 & 5.6 & 7.6 & 1.15 & $4.05 *$ & $2.65 *$ \\
\hline 30. Elettaria cardmomum Maton & Zingiberaceae & Sd. & Market & -- & 9.0 & 7.48 & 3.82 & 1.0 & 11.55 & 6.2 \\
\hline
\end{tabular}




\begin{tabular}{|c|c|c|c|c|c|c|c|c|c|c|}
\hline 31. & Embelia ribes Burm. f. & Fr. & Market & -- & 5.68 & 5.4 & 3.81 & 0.31 & 10.45 & 11.3 \\
\hline 32. & Emblica officinalis Gaertn. & Fr. & Narsapur & 10 & 6.77 & 3.85 & 3.29 & 0.31 & $10.45^{*}$ & $6.2 *$ \\
\hline 33. & Evolvulus alsinoides Linn. & Wh. Pl. & Narsapur & 57 & 5.93 & 6.95 & 14.6 & 7.19 & 7.75 & 4.4 \\
\hline 34. & Ferula foetida Regel & Exd. & Market & -- & 8.16 & 6.25 & 4.21 & 2.23 & $9.95 *$ & $9.35^{*}$ \\
\hline 35. & Glycyrrhiza glabra Linn. & Rt. & Market & -- & 6.31 & 5.29 & 8.14 & $4.93 *$ & $10.5 *$ & $7.05^{*}$ \\
\hline 36. & $\begin{array}{c}\text { Hedychium spicatum Ham. } \\
\text { ex Smith }\end{array}$ & Rz. & Market & -- & 8.12 & 8.09 & 4.19 & 0.72 & 9.85 & 4.5 \\
\hline 37. & Hemidesmus indicus $\mathrm{R} . \mathrm{Br}$. & Rt. & Narsapur & 65 & 8.85 & 6.94 & $11.28 *$ & $4.19 *$ & $6.7 *$ & $3.65^{*}$ \\
\hline 38. & Holarrhena antidysenterica Wall.Apocynaceae & St.bk. & Narsapur & 48 & 7.53 & 5.9 & 6.05 & 0.8 & 10.61 & $5.55^{*}$ \\
\hline 40. & Mucuna prurita Hook. & Sd. & Narsapur & 11 & 6.9 & 5.74 & $8.78 *$ & $2.84 *$ & $16.8 *$ & 4.05 \\
\hline 41. & Myristica fragrans Houtt. & Aril & Market & -- & 5.08 & 6.65 & 2.36 & 0.16 & 6.3 & 13.7 \\
\hline 42. & Myristica fragrans Houtt. & Sd. & Market & -- & 12.67 & 7.8 & 1.93 & 0.1 & $5.4 *$ & $8.8^{*}$ \\
\hline 43. & Nardostachys jatamansi DC. & Rt.+Rz. & Market & -- & 7.04 & 6.01 & $9.52 *$ & 4.41 & $2.95 * 2$ & 2.55 \\
\hline 44. & Nelumbo nucifera Gaertn. & Sd. & Market & -- & 7.29 & 7.72 & 3.93 & 0.06 & 12.9 & 4.8 \\
\hline 45. & Nymphea stellata Willd. & Fl. & Narsapur & 22 & 11.84 & 5.26 & $12.68 *$ & $3.12 *$ & $19.8 *$ & 3.65 \\
\hline 46. & Oryza sativa Linn. & Rt. & Narsapur & 15 & 3.97 & 5.2 & 7.96 & 3.59 & 5.9 & $4.1 *$ \\
\hline 47. & Phaseolus trilobus Ait. & $\mathrm{Pl}$. & Narsapur & 16 & 6.72 & 5.59 & $14.27 *$ & $6.27 *$ & $6.75 *$ & 5.6 \\
\hline 49. & $\begin{array}{r}\text { Picrorhiza kurroa } \text { Royle } \\
\text { ex. Benth. }\end{array}$ & Rz. & Market & -- & 5.99 & 6.48 & 4.62 & $1.57 *$ & 20.55 & 13.65 \\
\hline 50. & Piper cubeba Linn.f. & Fr. & Market & -- & 16.0 & 6.48 & $8.57 *$ & 0.68 & 17.2 & 14.6 \\
\hline 51. & Piper longum Linn. & Fr. & Market & -- & 11.25 & 6.98 & $7.28 *$ & 0.36 & 22 & 9.25 \\
\hline 52. & Piper nigrum Linn. & Fr. & Market & -- & 10.19 & 7.48 & $5.41 *$ & 0.32 & 6.9 & 6.1 \\
\hline 53. & Plumbago zeylanica Linn. & Rt. & Narsapur & 08 & 12.5 & 5 & $4.57 *$ & 0.36 & $11.75^{*}$ & 13.5 \\
\hline
\end{tabular}

Contd... 


\begin{tabular}{|c|c|c|c|c|c|c|c|c|c|c|c|}
\hline 54. & Prunus cerasoides D. Don & Rosaceae & St. & Market & -- & 5.6 & 6.24 & $10.08 *$ & 0.39 & 3.65 & $2.3 *$ \\
\hline 55. & Pterocarpus santalinus Linn. f. & Fabaceae & Ht.Wd. & Market & -- & 5.17 & 6.24 & 1.57 & 0.23 & 1.4 & 10.55 \\
\hline 56. & Pueraria tuberosa DC. & Leguminosae & Rt. & Market & -- & 6.43 & 8.08 & 2.26 & 0.17 & 25.8 & 4.6 \\
\hline 57. & Punica granatum Linn. & Punicaceae & Sd. & Narsapur & 32 & 7.04 & 4.5 & 3.55 & 0.13 & $32.4 *$ & 20.5 \\
\hline 58. & Rubia cordifolia Linn. & Rubiaceae & St. & Market & -- & 6.39 & 5.75 & $12.3 *$ & $0.7^{*}$ & 18.45 & 3.35 \\
\hline 59. & Saccharum spontaneum linn. & Gramineae & Rt. & Narsapur & 52 & 3.94 & 5.19 & $8.37 *$ & 1.97 & 4.2 & $2.5 *$ \\
\hline 60. & Santalum album Linn. & Santalaceae & Ht.Wd. & Narsapur & 50 & 6.92 & 5.95 & $1.09 *$ & 0.09 & 1.45 & 8.55 \\
\hline 61. & Scindapsus officinalis Schott & Araceae & Fr. & Market & -- & 9.08 & 8.06 & 5.21 & 0.44 & 29.45 & 4.8 \\
\hline 62. & $\begin{array}{c}\text { Sesbania bispinosa (Jacq.) } \\
\text { Fawett \& Rendle }\end{array}$ & Leguminosae & Rt. & Narsapur & 66 & 3.75 & 5.5 & 8.37 & 1.06 & 7.4 & 6.5 \\
\hline 63. & Sida cordifolia Linn. & Malvaceae & Rt. & Narsapur & 05 & 6.75 & 7.12 & 2.32 & 0.15 & 3.8 & 2.2 \\
\hline 64. & $\begin{array}{c}\text { Solanum xanthocarpum Schrad } \\
\text { \& Wendl. }\end{array}$ & Solanaceae & Rt. & Narsapur & 51 & 4.54 & 5.23 & 4.76 & 0.8 & 16.9 & 4.4 \\
\hline 65. & $\begin{array}{l}\text { Syzygium aromaticum (Linn.) } \\
\text { Merr.\&L.M.Perry }\end{array}$ & Myrtaceae & Fl. & Market & -- & 9.73 & 6.15 & 5.36 & 0.39 & 11.65 & 13.4 \\
\hline 66. & $\begin{array}{l}\text { Terminalia arjuna W. \& A. } \\
11.95^{*}\end{array}$ & Combretaceae & St. Bk. & Narsapur & 33 & 8.08 & 5.15 & 15.27 & $4.85^{*}$ & $10.5^{*}$ & \\
\hline 67. & Terminalia bellerica Roxb. & Combretaceae & Fr. & Narsapur & 31 & 6.79 & 4.6 & 3.25 & 0.29 & $33.4 *$ & 9.35 \\
\hline 69. & $\begin{array}{c}\text { Tinospora cordifolia (Willd.) } \\
\text { Miers. }\end{array}$ & Menispermaceae & St. & Narsapur & 09 & 8.2 & 3.54 & 11.46 & $3.88 *$ & $9.8 *$ & 3.15 \\
\hline 70. & Trachyspermum ammi Spargue. & Umbelliferae & Fr. & Market & -- & 8.78 & 5.65 & $9.69 *$ & $0.27 *$ & 15.7 & 6.6 \\
\hline 71. & Trapa bispinosa Roxb. & Onagraceae & Fr. & Market & -- & 12.59 & 7.03 & 2.34 & 0.13 & 8.65 & 1.85 \\
\hline 72. & Tribulus terrestris Linn & Zygophyllaceae & Fr. & Narsapur & 04 & 6.15 & 8.59 & 10.64 & 0.26 & 12.6 & 6.55 \\
\hline 73. & Vetiveria zizanioides(Linn.)Nash & Poaceae & Rt. & Narsapur & 21 & 7.71 & 5.66 & $11.13 *$ & 1.78 & 7.25 & 4.95 \\
\hline 74. & Withania somnifera Dunal & Solanaceae & Rt. & Market & -- & 8.42 & 5.58 & $9.05 *$ & 0.61 & $5.4 *$ & 12.5 \\
\hline 75. & Woodfordia fruticosa Kurz. & Lythraceae & Fl. & Narsapur & 07 & 35.6 & 4.67 & 6.23 & 0.69 & $25.8^{*}$ & 8.4 \\
\hline 76. & Zingiber officinale Rosc. & Zingiberaceae & Rz. & Market & -- & 8.68 & 7.17 & 4.99 & 1.41 & 10.25 & 5.05 \\
\hline
\end{tabular}

Where, 1- Place of collection; 2- Voucher specimen number; 3 - Moisture content; 4 - pH (1\% aqueous); 5 - Total ash; 6 - Acid insoluble ash; 7 - Water-soluble extractive; 8- Alcohol soluble extractive. * Values exceeding the Pharmacopeial limits. 
Table - 2. Results of phytochemical study

\begin{tabular}{|c|c|c|c|c|c|c|c|c|c|c|}
\hline S. No. Botanical name & Family & th used & 1 & 2 & 3 & 4 & 5 & 6 & 7 & 8 \\
\hline 1. Abutilon indicum (Linn.) Sw. & Malvaceae & Rt. & +ve & +ve & + ve & +ve & $+\mathrm{ve}$ & -ve & -ve & +ve \\
\hline 2. Acorus calamus Linn. & Araceae & Rz. & $+\mathrm{ve}$ & -ve & $+\mathrm{ve}$ & -ve & -ve & $+\mathrm{ve}$ & $+\mathrm{ve}$ & -ve \\
\hline 3. Aegle marmelos Corr. & Rutaceae & Fr. & $+\mathrm{ve}$ & $+\mathrm{ve}$ & $+\mathrm{ve}$ & $+\mathrm{ve}$ & $+\mathrm{ve}$ & $+\mathrm{ve}$ & $+\mathrm{ve}$ & + ve \\
\hline 4. Aegle marmelos Corr. & Rutaceae & St. Bk. & $+\mathrm{ve}$ & -ve & -ve & -ve & -ve & -ve & $+\mathrm{ve}$ & -ve \\
\hline 5. Aerva lanata (L.) Juss. ex. Schult & Amaranthaceae & Rt. & $+\mathrm{ve}$ & -ve & $+\mathrm{ve}$ & $+\mathrm{ve}$ & $+\mathrm{ve}$ & -ve & -ve & $+\mathrm{ve}$ \\
\hline 6. Ailanthes excelsa Roxb. & Simarubaceae & St. Bk. & $+\mathrm{ve}$ & -ve & -ve & -ve & $+\mathrm{ve}$ & -ve & -ve & -ve \\
\hline 7. Aloe vera Tourn.ex. Linn. & Liliaceae & Lf.Exd. & $+\mathrm{ve}$ & $+\mathrm{ve}$ & $+\mathrm{ve}$ & $+\mathrm{ve}$ & $+\mathrm{ve}$ & $+\mathrm{ve}$ & $+\mathrm{ve}$ & + ve \\
\hline 8. Alpinia galangaWilld. & Zingiberaceae & Rz. & $+\mathrm{ve}$ & $+\mathrm{ve}$ & + ve & + ve & $+\mathrm{ve}$ & $+\mathrm{ve}$ & -ve & $+\mathrm{ve}$ \\
\hline 9. Areca catechu Linn. ex. Benth. & Palmae & Sd. & $+\mathrm{ve}$ & $+\mathrm{ve}$ & $+\mathrm{ve}$ & $+\mathrm{ve}$ & $+\mathrm{ve}$ & $+\mathrm{ve}$ & $+\mathrm{ve}$ & $+v e$ \\
\hline 10. Asparagus racemosus Willd. & Liliaceae & $\mathrm{Rt}$ & -ve & -ve & $+\mathrm{ve}$ & -ve & $+\mathrm{ve}$ & $+\mathrm{ve}$ & -ve & -ve \\
\hline 11. Asteracantha longifolia Nees & Acanthaceae & Rt. & $+\mathrm{ve}$ & -ve & $+\mathrm{ve}$ & -ve & $+\mathrm{ve}$ & $+\mathrm{ve}$ & $+\mathrm{ve}$ & -ve \\
\hline 12. Azadirachta indica A. Juss & Meliaceae & St. Bk. & $+\mathrm{ve}$ & $+\mathrm{ve}$ & $+\mathrm{ve}$ & $+\mathrm{ve}$ & $+\mathrm{ve}$ & $+\mathrm{ve}$ & $+\mathrm{ve}$ & $+\mathrm{ve}$ \\
\hline 13. Bacopa monnieri (Linn.) Pennel & Scorphulariaceae & Pl. & $+\mathrm{ve}$ & $+\mathrm{ve}$ & + ve & + ve & $+\mathrm{ve}$ & -ve & $+\mathrm{ve}$ & + ve \\
\hline 14. Bambusa arundinacea Willd. & Gramineae & S.C. & -- & -- & -- & -- & -- & -- & -- & -- \\
\hline 15. Berberis aristata DC. & Berberidaceae & St. & $+\mathrm{ve}$ & -ve & -ve & $+\mathrm{ve}$ & $-\mathrm{ve}$ & -ve & -ve & $+\mathrm{ve}$ \\
\hline 16. Boerhaavia diffusa Linn. & Nyctaginaceae & Wh. Pl. & $+\mathrm{ve}$ & -ve & -ve & -ve & -ve & -ve & $+\mathrm{ve}$ & -ve \\
\hline 17. Carum carvi Linn. & Umbelliferae & Fr. & $+\mathrm{ve}$ & $+\mathrm{ve}$ & + ve & + ve & $+\mathrm{ve}$ & $+\mathrm{ve}$ & -ve & + ve \\
\hline 18. Cassia fistula Linn. & Leguminosae & Fr.P & $+\mathrm{ve}$ & -ve & $+\mathrm{ve}$ & $+\mathrm{ve}$ & $+\mathrm{ve}$ & -ve & $+\mathrm{ve}$ & +ve \\
\hline 19. Centella asiatica (L.) Urban & Apiaceae & Wh. Pl. & $+\mathrm{ve}$ & $+\mathrm{ve}$ & $+\mathrm{ve}$ & $+\mathrm{ve}$ & $+\mathrm{ve}$ & -ve & $+\mathrm{ve}$ & $+\mathrm{ve}$ \\
\hline $\begin{array}{c}\text { 20. Cinnamomum camphora Nees } \\
\& \text { Eberm }\end{array}$ & Lauraceae & Vol. Matter & -- & -- & -- & -- & -- & -- & -- & -- \\
\hline $\begin{array}{l}\text { 21. Cinnamomum tamala Nees } \\
\& \text { Eberm }\end{array}$ & Lauraceae & Lf. & $+\mathrm{ve}$ & $+\mathrm{ve}$ & $+\mathrm{ve}$ & $+\mathrm{ve}$ & $+\mathrm{ve}$ & -ve & $+\mathrm{ve}$ & +ve \\
\hline 22. Cinnamomum zeylanicum Blume & Lauraceae & St. Bk. & -ve & $+\mathrm{ve}$ & $+\mathrm{ve}$ & $+\mathrm{ve}$ & $+\mathrm{ve}$ & $+\mathrm{ve}$ & $+\mathrm{ve}$ & $+\mathrm{ve}$ \\
\hline 23. Commiphora myrrha (Nees)Engl.B & Burseraceae & Exd. & -ve & $+\mathrm{ve}$ & + ve & + ve & $+\mathrm{ve}$ & $+\mathrm{ve}$ & $+\mathrm{ve}$ & $+\mathrm{ve}$ \\
\hline 24. Coriandrum sativum Linn. & Umbelliferae & Fr. & -ve & + ve & + ve & + ve & $+\mathrm{ve}$ & $+\mathrm{ve}$ & + ve & +ve \\
\hline
\end{tabular}

Contd... 


\begin{tabular}{|c|c|c|c|c|c|c|c|c|c|c|c|}
\hline 25. & Cuminum cyminum Linn. & Umbelliferae & Fr. & $+\mathrm{ve}$ & $+\mathrm{ve}$ & $+\mathrm{ve}$ & $+\mathrm{ve}$ & $+\mathrm{ve}$ & $+\mathrm{ve}$ & $+\mathrm{ve}$ & $+\mathrm{ve}$ \\
\hline 26. & Curcuma longa Linn. & Zingiberaceae & Rz. & + ve & + ve & + ve & $+\mathrm{ve}$ & -ve & + ve & +ve & + ve \\
\hline 27. & Cymbopogan citratus (DC.)Stap & Gramineae & Rt. & + ve & -ve & +ve & $+\mathrm{ve}$ & -ve & -ve & +ve & + ve \\
\hline 28. & Cyperus rotundus Linn. & Cyperaceae & Rz. & -ve & $+\mathrm{ve}$ & -ve & $+\mathrm{ve}$ & -ve & $+\mathrm{ve}$ & + ve & $+\mathrm{ve}$ \\
\hline 29. & Desmostachya bipinnata Stapf. & Gramineae & Rt. & + ve & -ve & +ve & -ve & -ve & +ve & -ve & -ve \\
\hline 30. & Elettaria cardmomum Maton & Zingiberaceae & Sd. & $+\mathrm{ve}$ & -ve & -ve & $-\mathrm{ve}$ & $+\mathrm{ve}$ & $+\mathrm{ve}$ & + ve & -ve \\
\hline 31. & Embelia ribes Burm. f. & Myrsinaceae & Fr. & $+\mathrm{ve}$ & -ve & $+\mathrm{ve}$ & $+\mathrm{ve}$ & -ve & $+\mathrm{ve}$ & $+\mathrm{ve}$ & $+\mathrm{ve}$ \\
\hline 32. & Emblica officinalis Gaertn. & Euphorbiaceae & Fr. & $+\mathrm{ve}$ & + ve & -ve & $+\mathrm{ve}$ & $+\mathrm{ve}$ & $+\mathrm{ve}$ & + ve & $+\mathrm{ve}$ \\
\hline 33. & Evolvulus alsinoides Linn. & Convolvulaceae & Wh. Pl. & $+\mathrm{ve}$ & + ve & + ve & $+\mathrm{ve}$ & $+\mathrm{ve}$ & -ve & + ve & $+\mathrm{ve}$ \\
\hline 34. & Ferula foetida Regel & Umbellifereae & Exd. & -ve & -ve & + ve & -ve & -ve & $+\mathrm{ve}$ & + ve & -ve \\
\hline 35 . & Glycyrrhiza glabra Linn. & Fabaceae & Rt. & -ve & + ve & + ve & $+\mathrm{ve}$ & $+\mathrm{ve}$ & + ve & + ve & $+\mathrm{ve}$ \\
\hline 36. & $\begin{array}{c}\text { Hedychium spicatum Ham. } \\
\text { ex Smith }\end{array}$ & Zingiberaceae & Rz. & -ve & -ve & $+\mathrm{ve}$ & -ve & $+\mathrm{ve}$ & $+\mathrm{ve}$ & $+\mathrm{ve}$ & -ve \\
\hline 37. & Hemidesmus indicus $\mathrm{R} . \mathrm{Br}$. & Asclepiadaceae & Rt. & $+\mathrm{ve}$ & -ve & $+\mathrm{ve}$ & $+\mathrm{ve}$ & $+\mathrm{ve}$ & -ve & + ve & $+\mathrm{ve}$ \\
\hline 38. & Holarrhena antidysenterica Wal & Apocynaceae & St.bk. & + ve & -ve & + ve & $+\mathrm{ve}$ & $+\mathrm{ve}$ & + ve & +ve & + ve \\
\hline 39. & Mesua ferrea Linn. & Clusiaceae & Fl. & $+\mathrm{ve}$ & + ve & -ve & $+\mathrm{ve}$ & $+\mathrm{ve}$ & $+\mathrm{ve}$ & + ve & $+\mathrm{ve}$ \\
\hline 40. & Mucuna prurita Hook. & Leguminosae & Sd. & $+\mathrm{ve}$ & -ve & + ve & $+\mathrm{ve}$ & $+\mathrm{ve}$ & $+\mathrm{ve}$ & + ve & $+\mathrm{ve}$ \\
\hline & Myristica fragrans Houtt. & Myristicaceae & Aril & -ve & -ve & + ve & $+\mathrm{ve}$ & $+\mathrm{ve}$ & $+\mathrm{ve}$ & + ve & + ve \\
\hline & Myristica fragrans Houtt. & Myristicaceae & Sd. & $+\mathrm{ve}$ & -ve & -ve & $+\mathrm{ve}$ & $+\mathrm{ve}$ & + ve & -ve & $+\mathrm{ve}$ \\
\hline & Nardostachys jatamansi DC. & Valerianaceae & Rt.+Rz. & + ve & -ve & $+\mathrm{ve}$ & -ve & $+\mathrm{ve}$ & -ve & +ve & -ve \\
\hline & Nelumbo nucifera Gaertn. & Nymphaeaceae & Sd. & + ve & -ve & -ve & $+\mathrm{ve}$ & $+\mathrm{ve}$ & + ve & -ve & + ve \\
\hline & Nymphea stellata Willd. & Nymphaeaceae & Fl. & $+\mathrm{ve}$ & + ve & + ve & $+\mathrm{ve}$ & $+\mathrm{ve}$ & -ve & + ve & $+\mathrm{ve}$ \\
\hline 46. & Oryza sativa Linn. & Gramineae & Rt. & $+\mathrm{ve}$ & -ve & + ve & -ve & $+\mathrm{ve}$ & -ve & + ve & -ve \\
\hline 47. & Phaseolus trilobus Ait. & Leguminosae & Pl. & + ve & +ve & +ve & $+\mathrm{ve}$ & $+\mathrm{ve}$ & -ve & +ve & + ve \\
\hline & $\begin{array}{c}\text { Phyllanthus amarus Schum } \\
\& \text { Thonn. }\end{array}$ & Euphorbiaceae & Pl. & $+\mathrm{ve}$ & $+\mathrm{ve}$ & $+\mathrm{ve}$ & $+\mathrm{ve}$ & $+\mathrm{ve}$ & -ve & -ve & + ve \\
\hline & $\begin{array}{r}\text { Picrorhiza kurroa Royle } \\
\text { ex. Benth. }\end{array}$ & Scrophulariaceae & Rz. & -ve & -ve & $+\mathrm{ve}$ & $+\mathrm{ve}$ & -ve & $+\mathrm{ve}$ & -ve & + ve \\
\hline 50. & Piper cubeba Linn.f. & Piperaceae & Fr. & + ve & -ve & + ve & $+\mathrm{ve}$ & -ve & + ve & -ve & + ve \\
\hline & Piper longum Linn. & Piperaceae & Fr. & +ve & +ve & -ve & $+\mathrm{ve}$ & -ve & +ve & +ve & +ve \\
\hline 52. & Piper nigrum Linn. & Piperaceae & Fr. & $+\mathrm{ve}$ & + ve & + ve & $+\mathrm{ve}$ & -ve & $+\mathrm{ve}$ & + ve & $+\mathrm{ve}$ \\
\hline
\end{tabular}

Contd... 


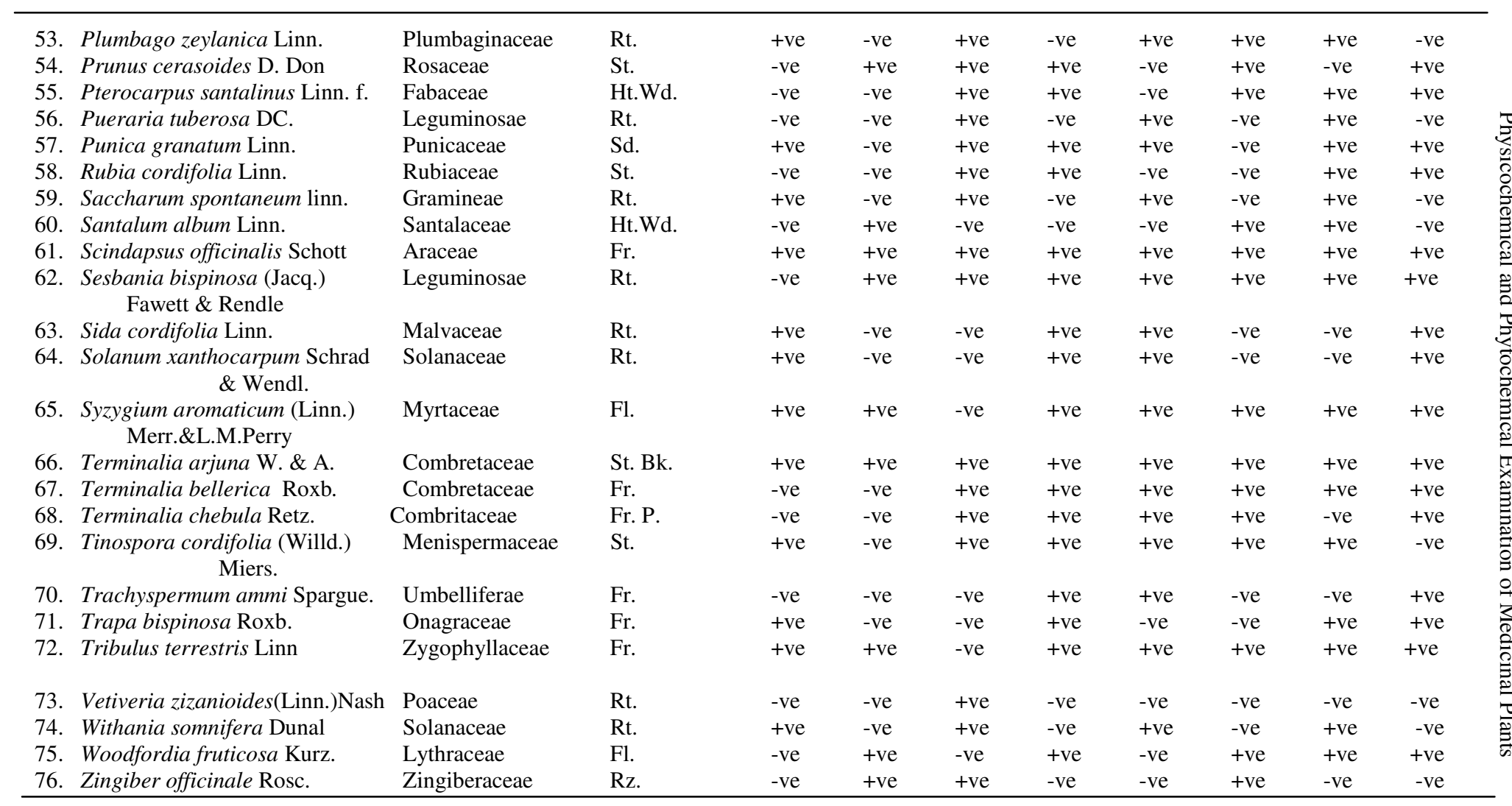

Where, 1 - Alkaloid; 2 - Flavonoid; 3 - Glycoside; 4 - Phenol; 5 - Saponin; 6 - Resin; 7 - Steroid; 8 - Tannin. 


\section{References}

1. Karnick C R, Nagarjun 1978, 5, 26-32.

2. Joshi M C and Sabnis S D, B ME B R 1989, 10(1-2), 61-82.

3. Puri H S, B MEBR 1980, 1(3), 384-392.

4. Agarwal R G, Pant P, Tiwari L C, Singh J, Pandey M J and Tiwary, D. N. B M E B R 1989, 10(3-4), 176-186.

5. Tewari L C, Sanwal P, Singh J and Joshi P, B ME B R 1984, 5(1-2), 71-81.

6. Ashok K S, B ME B R 1988, 9(1-2), 68-77.

7. Ayurvedic Pharmacopoeia of India, Dept. of ISM\&H, Ministry of Health and Family Welfare: New Delhi, 1989; Vol.-I.

8. Ayurvedic Pharmacopoeia of India, Dept. of ISM\&H, Ministry of Health and Family Welfare: New Delhi, 1999; Vol.-II.

9. Ayurvedic Pharmacopoeia of India, Dept. of ISM\&H, Ministry of Health and Family Welfare: New Delhi, 2001; Vol.-III.

10. Ayurvedic Pharmacopoeia of India, Dept. of ISM\&H, Ministry of Health and Family Welfare: New Delhi, 2004; Vol.-IV.

11. Pullaiah T, Prabhakar C and Raviprasad Rao B, Flora of Medak District (Andhra Pradesh); Daya Publishing House: Delhi, 1998.

12. Narasimha Rao, G. Studies on the floristics of angiosperms and the dermotypes of Acanthaceae of Narsapur taluk (Medak district, Andhra Pradesh); Ph. D. thesis, Osmania University: Hyderabad, 1986.

13. Quality Control Methods for Medicinal Plant Materials; WHO: Geneva, 1998.

14. Pharmacopoeial Standards for Ayurvedic Formulations; CCRAS: New Delhi, 1987.

15. Trease, G E, Evans W C Pharmacognosy; $11^{\text {th }}$ Ed.; Bailliere Tindall: London, 1978.

16. Wallis, T. E. Textbook of Pharmacognosy; $5^{\text {th }}$ Ed.; J \& A Churchill Ltd.: London, 1967.

17. Alam M, Dasan K K S, Rukmani B and Rao R B, Journal of Research in Ayurveda and Siddha 1990, 11(1-4): 56-60. 


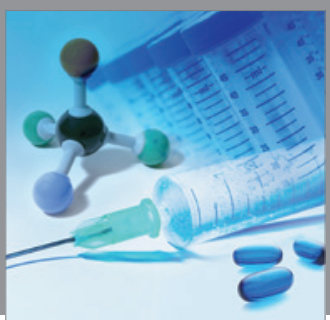

International Journal of

Medicinal Chemistry

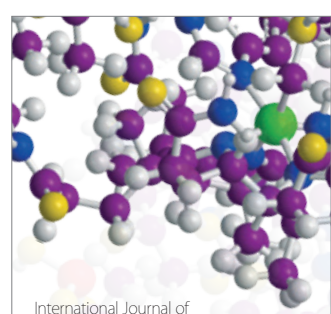

Carbohydrate Chemistry

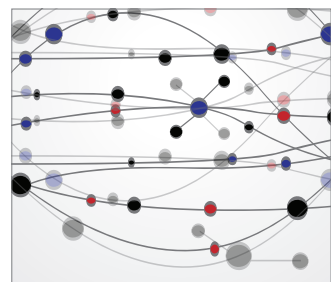

The Scientific World Journal
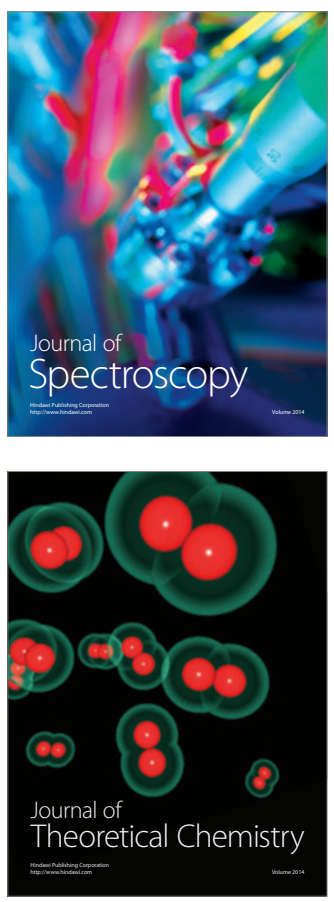
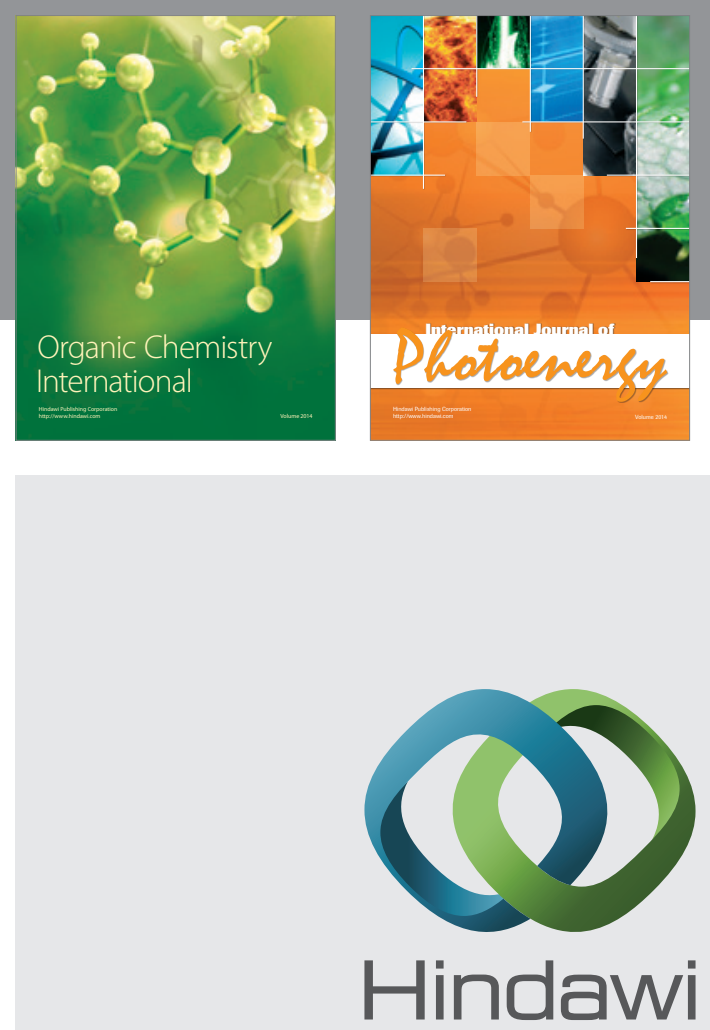

Submit your manuscripts at

http://www.hindawi.com
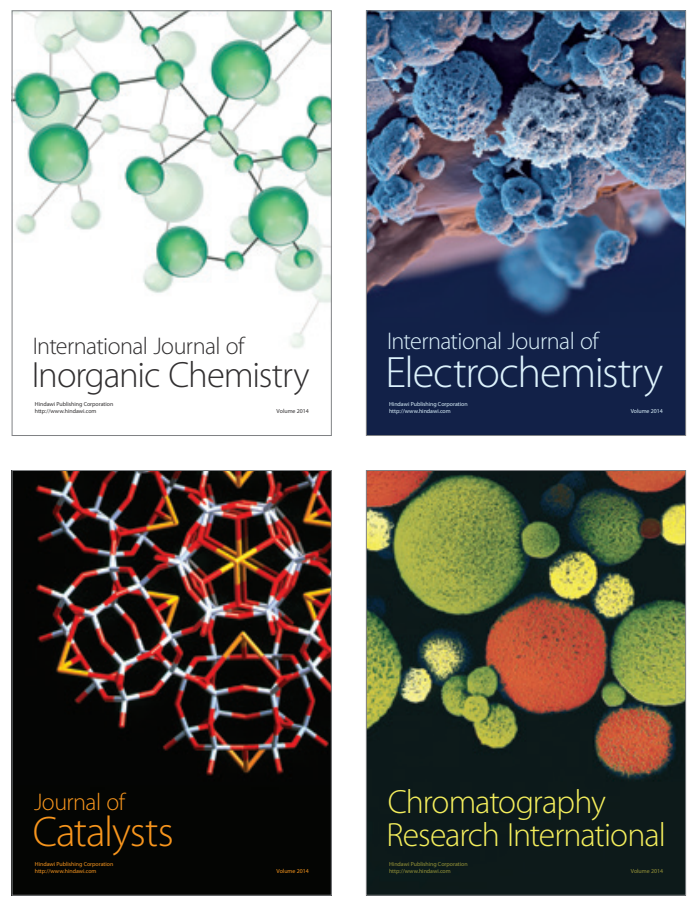
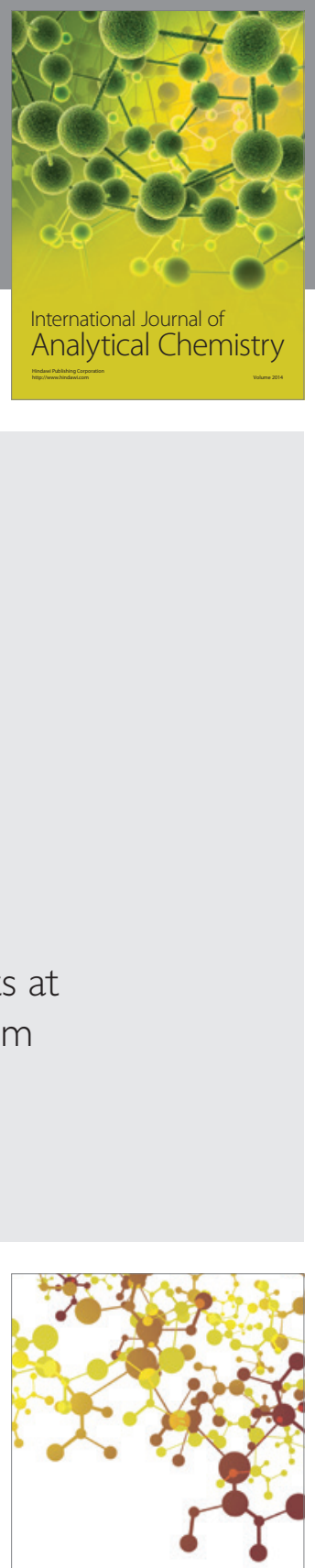

Journal of

Applied Chemistry
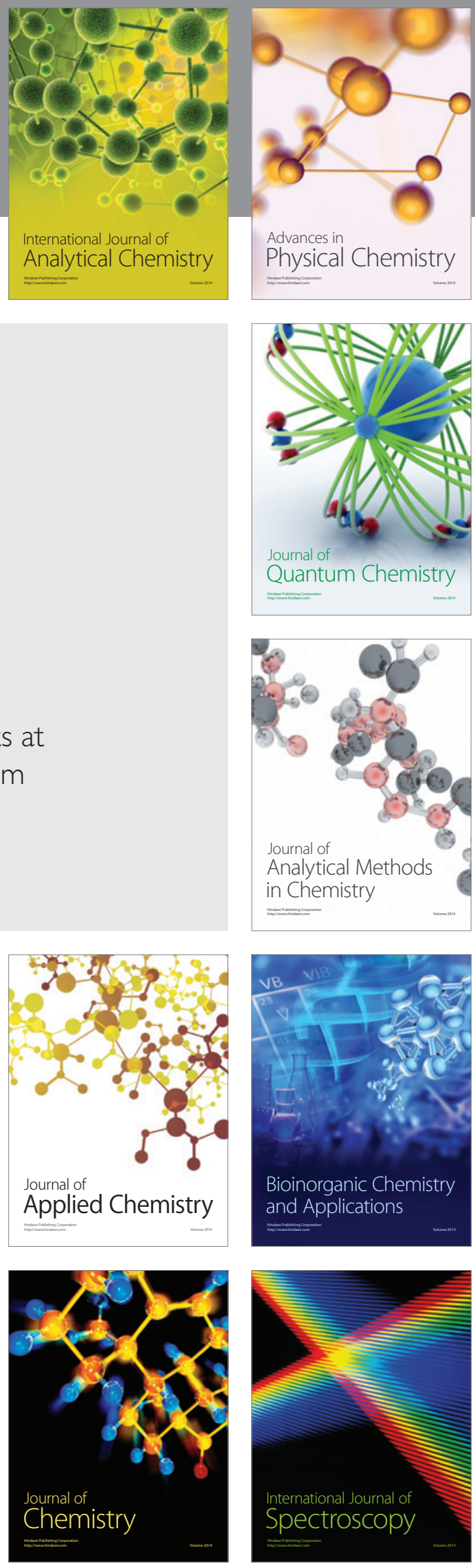